\title{
A self-help version of the New Forest Parenting Programme for parents of children with attention deficit hyperactivity disorder: a qualitative study of parent views and acceptability
}

\author{
Joanne $\operatorname{Tarver}^{1}{ }^{1}$, David Daley ${ }^{2}$ \& Kapil Sayal ${ }^{2}$ \\ ${ }^{1}$ School of Psychology, College of Health and Life Sciences, Aston University, Birmingham, UK \\ ${ }^{2}$ Division of Psychiatry and Applied Psychology, School of Medicine, University of Nottingham, Nottingham, UK
}

\begin{abstract}
Background: Although parent interventions are recommended as a frontline treatment approach for children with attention deficit hyperactivity disorder (ADHD), a number of practical and situational barriers can impact accessibility and availability. Self-help parent interventions offer a potential alternative to therapist-led interventions when barriers prevent access to face-to-face treatment. This qualitative study aims to explore participant views and acceptability of self-help parent interventions. Method: Semi-structured interviews were conducted with parents/carers of 12 children (age 6-10 years) with ADHD who received the self-help version of the New Forest Parenting Programme (NFPP-SH) as part of a randomised controlled trial. Thematic analysis (as proposed by Braun and Clarke) was used to analyse the data. Results: Overall, participants had favourable views of the self-help intervention but also experienced some barriers to treatment adherence. Six key themes were identified in parent interviews related to parental desire to learn more; acquisition of new skills; the flexibility of the intervention; self-help intervention vs. traditional therapist-led formats; barriers to engagement in the home environment; and need for earlier access to help. Conclusions: NFPP-SH was an acceptable intervention for parents. However, some parents may need additional support to overcome barriers associated with completing an intervention at home. Implications for healthcare providers and researchers developing selfhelp parent interventions for ADHD and child behaviour are discussed.
\end{abstract}

\section{Key Practitioner Message}

- Self-help parent interventions are known to be effective for reducing parent-reported child behaviour problems; effect sizes for parent-reported outcomes may be comparable to therapist-led intervention.

- To our knowledge, this is the first study to use qualitative methods to explore participant views and acceptability of self-help parent interventions for children with attention deficit hyperactivity disorder (ADHD).

- Our findings suggest that parents had favourable views of the self-help version of the New Forest Parenting Programme (NFPP-SH).

- Clinicians and service commissioners may wish to consider offering NFPP-SH to parents of children with ADHD, especially soon after diagnosis when parents may be seeking additional information, or where barriers to face-to-face treatment exist.

Keywords: ADHD; qualitative methods; parent training

\section{Introduction}

Parent interventions are effective treatments for behavioural problems in young children with attention deficit hyperactivity disorder (ADHD) and are recommended as a frontline treatment approach (NICE, 2018; Tarver, Daley, \& Sayal, 2014). However, a number of barriers can prevent access to traditional therapist-led intervention. This includes the costs associated with therapistled intervention which can impact service provision (Foster, Johnson-Shelton, \& Taylor, 2007) and situational and practical barriers (e.g. transport/child care) limiting parental ability to attend sessions (Smith et al., 2015). Access to traditional parent-led intervention could also be particularly challenging during pandemics, whilst physical distancing measures are in place (Cortese et al., 2020). Self-help parent interventions, where parents are provided with materials to teach intervention components to themselves, might provide a suitable alternative. Self-help interventions are available as manuals, online courses or via apps for mobile phone and tablet use; some interventions also offer brief therapist support to guide the parent through the programme (Tarver, Daley, Lockwood, et al., 2014).

There is evidence that self-help parent interventions are effective in reducing parent-reported externalising child behaviour (Dose et al., 2017; Tarver, Daley, Lockwood, et al., 2014). When supplemented with therapist input over the telephone, intervention effects may be maintained at 12 months following intervention (Ise,

(c) 2021 The Authors. Child and Adolescent Mental Health published by John Wiley \& Sons Ltd on behalf of Association for Child and Adolescent Mental Health

This is an open access article under the terms of the Creative Commons Attribution License, which permits use, distribution and reproduction in any medium, provided the original work is properly cited. 
Kierfeld, \& Döpfner, 2015). For ADHD management, a self-help version of the New Forest Parenting Programme (NFPP) has been developed. The NFPP is a behavioural parent intervention developed specifically for ADHD; therapist-led NFPP has been shown to be effective for the treatment of preschool ADHD and behaviour problems in a number of randomised controlled trials (RCTs) (Lange et al., 2018; Shimabukuro et al., 2017; Sonuga-Barke et al., 2018). The self-help version of the NFPP (NFPP-SH) has been trialled in a small-scale RCT and was associated with improvements in parent-reported child ADHD symptoms and parent efficacy and satisfaction (Daley \& O'Brien, 2013).

Despite the importance of using quantitative measures to assess intervention outcome, the need for qualitative methods in intervention research has also been stressed (Campbell, 2000). One example is the use of nested qualitative interview methods within a trial, such as the exploration of parent views on intervention acceptability following their involvement in the trial. Such methods provide an opportunity to complement quantitative findings, gain information about participant experience and capture explanations for main trial outcomes (Lewin, Glenton, \& Oxman, 2009). If self-help interventions are to be considered a viable alternative to traditional therapist-led intervention, exploration of participant views of self-help interventions is needed. Whilst treatment acceptability is related to treatment outcome, the relationship is modest; therapeutic change may not, therefore, indicate treatment acceptability and vice versa (Kazdin \& Wassell, 2000). Self-help interventions are a potential costeffective alternative to overcome barriers to therapistled interventions. However, they may still be burdensome for families, or other factors might impact parent engagement. This highlights the importance of supplementing investigations of therapeutic change with explorations of participant experience and acceptability using qualitative methods. Indeed, qualitative interview and focus group studies have highlighted how psychological and situational barriers to intervention, such as work commitments and fear of attending sessions, can impact engagement in group-based parent interventions in ADHD (Smith et al., 2015; Taylor et al., 2015). However, to our knowledge, there are no published qualitative studies that have assessed parental views of self-help parent interventions.

This qualitative study was conducted following a small-scale RCT of the NFPP-SH when used in adjunct to usual treatment (see Daley, Tarver, \& Sayal, 2020). Findings from the RCT suggested that NFPP-SH may have beneficial effects for parental efficacy and negative comments made by parents during a recorded speech sample. However, no additional effect on child behaviour was found. The current study describes interviews conducted with participants who received NFPP-SH as part of the RCT. Main trial outcomes are reported in detail elsewhere (Daley et al., 2020). Specifically, this study had two aims: (a) to explore participants' experience of NFPP-SH and their views on how it may compare to traditional therapist-led interventions, and (b) to explore potential barriers and facilitators of adherence to guide the development of future self-help interventions.

\section{Methods}

This nested qualitative study uses interview data collected as part of a small-scale RCT of the NFPP-SH (Daley et al., 2020 ClinicalTrials.gov identifier: NCT02174952). The aim of the RCT was to explore the effectiveness of NFPP-SH when used in adjunct to a child's usual treatment, including pharmacotherapy. Fifty-two families took part in the RCT; 28 received the NFPP-SH in adjunct to their usual care (TAU+SH), whilst 24 were allocated to usual treatment alone (TAU). Those allocated to $\mathrm{TAU}+\mathrm{SH}$ received an intervention pack containing an introductory DVD and the NFPP-SH manual (Laver-Bradbury, Thompson, Weeks, Daley, \& Sonuga-Barke, 2010) consisting of two parts: (a) psychoeducation, and (b) a six-step programme including empirically supported behavioural management strategies. The programme also includes ideas for parent-led games and activities aimed at targeting self-regulatory and executive function deficits often present in children with ADHD (Sonuga-Barke et al., 2018). This study received ethical approval from the NHS Research Ethics Committee (REC ref: 12/EM/0200).

\section{Participants}

Participants were 12 parents who took part in a small-scale RCT of NFPP-SH and were allocated to the intervention arm (see Daley et al., 2020). Families were recruited into the RCT from 11 participating community paediatric and child and adolescent mental health clinics throughout England. Participating services were those providing diagnostic and mental health services for young children with ADHD. Families of children aged 6-10 years were eligible for the trial if they had received a clinical diagnosis of ADHD and were in receipt of medication for $\mathrm{ADHD}$, and their parent/caregiver was aged 18 years or over. Families were not eligible if parents were unable to read English, or if the referring clinician felt that the parent/caregiver would be unable to complete the intervention (e.g. parent had mental health difficulties). Clinicians working in participating clinics approached eligible families to complete expression of interest (EOI) forms. EOI forms were passed to the research team who contacted parents to provide information about the study and obtain consent. For further information about recruitment into the main RCT, intervention content and procedures see Daley et al., 2020.

Of the 28 parents who received the self-help intervention as part of the RCT, six dropped out or were unable to be contacted for postintervention assessments, four declined to take part in the nested qualitative interview study, and the researcher was unable to contact six parents to conduct the interview. Semistructured interviews were conducted with 12 recipients of the NFPP-SH within one month of the end of the intervention period. Results from these 12 interviews are described in this current study (see Table 1 for participant demographics). To assess whether the 12 participants differed from those who did not take part in the nested qualitative interview, a series of comparison tests were run. Parents in this nested qualitative sample reported having fewer people that they could rely on to help with the care of their child $(t[26]=2.09, p<.05)$ and fewer symptoms of low mood on the General Health Questionnaire (GHQ12; Goldberg \& Williams, 1988) $t[26]=2.24, p<.05$ ). Parents who completed qualitative interviews also perceived lower levels of child stigma associated with ADHD medication $(U=51.0$, $p<.05)$.

\section{Procedure}

Following their involvement in the trial, all recipients of the NFPP-SH who completed postintervention follow-up assessments were invited to take part in the nested qualitative study. Interviews took place over the telephone, with the first author, within one month of the end of the intervention period. Interviews were recorded and transcribed verbatim. Participants were asked to be alone in a quiet room for the duration of the interview. The interview aimed to elicit parents' feelings about their experience of the self-help intervention and its acceptability and their reasons for taking part in the study. The 
Table 1. Participant demographics

\begin{tabular}{|c|c|c|}
\hline \multirow[t]{2}{*}{ Child age (years) } & Mean $(S D)$ & $8.34(1.45)$ \\
\hline & Min-max & $6-10$ \\
\hline \multirow[t]{2}{*}{ Child sex; $n(\%)$} & Male & $9(75 \%)$ \\
\hline & Female & $3(25 \%)$ \\
\hline Child ethnicity; $n$ (\%) & White British & $12(100 \%)$ \\
\hline Child diagnosis; $n$ (\%) & $\begin{array}{l}\text { Attention deficit hyperactivity } \\
\text { disorder (ADHD) }\end{array}$ & $12(100 \%)$ \\
\hline \multirow[t]{4}{*}{$\begin{array}{l}\text { Additional } \\
\text { diagnoses; } n(\%)\end{array}$} & $\begin{array}{l}\text { Oppositional Defiant Disorder } \\
\text { (ODD) }\end{array}$ & $1(8 \%)$ \\
\hline & $\begin{array}{l}\text { Autism Spectrum Disorder } \\
\text { (ASD) }\end{array}$ & $1(8 \%)$ \\
\hline & $\begin{array}{l}\text { Generalised Anxiety Disorder } \\
\text { (GAD) }\end{array}$ & $1(8 \%)$ \\
\hline & Attachment Disorder & $1(8 \%)$ \\
\hline \multirow{2}{*}{$\begin{array}{l}\text { Parent/carer age; } \\
\text { mean }(S D)\end{array}$} & Mean $(S D)$ & 40.17 \\
\hline & Min-max & $29-52$ \\
\hline $\begin{array}{l}\text { Parent/carer sex; } \\
n(\%)\end{array}$ & Female & $12(100 \%)$ \\
\hline \multirow{5}{*}{$\begin{array}{l}\text { Highest level of } \\
\text { parent education }\end{array}$} & No formal qualifications & $1(8 \%)$ \\
\hline & Completed school/GCSE's & $4(33 \%)$ \\
\hline & $\begin{array}{l}\text { Completed college/Further } \\
\text { education/A-Levels }\end{array}$ & $4(33 \%)$ \\
\hline & $\begin{array}{l}\text { Undergraduate Degree/ } \\
\text { Higher Education }\end{array}$ & 0 \\
\hline & Postgraduate Degree & $3(25 \%)$ \\
\hline
\end{tabular}

interviewer had spoken to parents on several occasions previously, in order to invite them to take part in the RCT, gain informed consent and collect outcome data. The participants were therefore aware of the interviewer's involvement in the study. However, parents were asked to answer questions as honestly as possible and in as much detail as they could. An interview schedule (see Appendix S1) was developed containing open-ended questions to address the aims of the research. As the first qualitative examination of NFPP-SH, interview questions were developed by the first author and were further refined during research team meetings involving researchers with extensive experience in parent-led intervention development and trials. Due to the small-scale nature of this study, a pilot study to test the interview schedule was not conducted. After the first two interviews, obtained data were reviewed to ensure that sufficient data were being obtained. This revealed that parents did not offer information about any potential impact of receiving the intervention on family life; the schedule was modified in order to capture this information. A qualitative interview schedule should be allowed to evolve throughout data collection to ensure that sufficient data are being obtained (Braun \& Clarke, 2013). The final schedule consisted of approximately seven open-ended questions. Parents were also asked three structured questions to explore their views on intervention acceptability. These questions asked parents whether they would recommend the intervention to a friend, would have liked to have been offered the intervention as part of their child's care and whether they think the intervention should be routinely offered to parents.

\section{Analysis}

Thematic analysis was used to code and analyse the data. This was chosen as the most appropriate form of methodology as it allows for the analysis of both inductive (emerging directly from the data) and deductive (derived from theory) themes. This approach allows for flexibility within the analysis to identify themes central in answering the research question and account for naturally occurring themes within the dataset (Braun \& Clarke, 2013). Further, thematic analysis is a robust, yet accessible, methodology widely used in applied health research including parent intervention research in relation to ADHD (Braun \& Clarke, 2014; Taylor et al., 2015). The analysis followed the six-stage approach to thematic analysis proposed by Braun \& Clarke (Braun \& Clarke, 2006), including (a) all transcripts were read thoroughly to obtain familiarisation with the data; (b) data were coded and highlighted and codes were placed into meaningful groups. Codes were initially placed into deductively derived groups that related to the aims of the research (parent views and acceptability of the intervention, including in comparison to therapist-led formats, and perceived barriers to the intervention); (c) themes and subthemes within the coded data were identified; (d) themes were reviewed to ensure clear distinction and identify how they fit together; (e) themes were defined and names given to themes; (f) the analysis was related back to study aims and quotes were selected which provided the most compelling example of the theme. Analysis was conducted by hand by the first author. Themes and included codes were discussed between all authors in team meetings before the analysis was finalised to ensure findings were not restricted to the views of a single author.

\section{Results}

The analysis revealed that data from parental interviews fit into six key themes: (a) parental desire to learn more; (b) acquisition of new skills; (c) the flexibility of the intervention; (d) the self-help intervention versus traditional therapist-led formats; (e) barriers to engagement in the home environment; and (f) earlier access to help.

\section{Parental desire to learn more}

In response to a question about their reasons for participating in the research, parents expressed a desire to learn more. This included a desire to understand ADHD itself, and strategies to help manage their child's behaviour. This theme emerged despite many parents reporting that they had attended a parenting programme previously. Some parents also reported previous attempts to research the condition themselves. Despite this, it was evident that parents were keen to engage in other opportunities to enhance their knowledge.

I didn't fully understand what the ADHD was, I mean I did google it, but in the book itself it explained a lot more about why they do what they do and what you can do better... so I could up my knowledge and do whatever I could to help them more and to bond more as a family. Parent 2

Some of the parents commented that previous programmes that they had participated in were not specific to ADHD, and consequently, this self-help intervention gave them a better understanding of ADHD and how it impacts child behaviour. Parents therefore valued the psychoeducation component of the intervention. Because of this increased awareness, some parents stated that they developed a more empathic approach towards their child.

[I gained] 'more of an understanding, which makes, I think me as a parent, more patient towards him'. Parent 5

\section{Acquisition of new skills}

Parents discussed some of the new techniques that they had acquired as a direct result of engaging in the intervention. This included setting aside playtime every day, engaging in memory games, reward charts and visual cues to support their child's organisational skills. Generally, the skills that parents discussed as being beneficial 
were techniques that may be more specific for children with ADHD. Parents also often described these techniques as ones that they had not encountered before, perhaps because the interventions that they had previously attended were not specifically targeted for children with ADHD.

The memory section with playing the games and stuff, I would never have thought of doing that, the main thing it's obviously helping them with their memory but it's giving you good quality time together as well. Parent 1

Whilst parents acknowledged the benefits of ADHD specific techniques for their child's organisational skills, there was also a sense that parents appreciated the opportunity that the new techniques gave them to spend more time with their child, have some fun with them and possibly improve the parent-child relationship.

I drew pictures of the toothbrush and the toothpaste and the pictures of him washing his face.... and he thought it was hysterical because Mummy can't draw and it's nice that he laughed about it, because we did have a laugh about it. Parent 9

It was anticipated that parents may have encountered some of the behavioural strategies included in NFPP-SH before. Whilst all of the parents stated that they already knew some of the strategies for child behaviour (e.g. time out), parents also spoke positively about using the book as a reminder of some of the strategies that they could be using but had forgotten. One parent, who reported a more passive engagement in the intervention, also stated that having the book reminded them that their child needs to be parented differently to other children.

I've got so used to telling them off all the time and grounding them, that I had forgotten that they are children and I need to reassure them and make them know that I still care. Parent 2

\section{The flexibility of the intervention: the when, the where and the how}

In terms of intervention delivery, parents spoke positively about the flexibility of the intervention and their ability to complete it at a time and place most convenient to them. Parents could fit their involvement in this intervention into their normal, everyday routine. Some of the parents discussed this in comparison to having to keep appointments and take time off work to attend therapistled sessions.

It is like having a therapy session but you've got it in a book and you can do it at your own time, you know, effort, when you want to read it, whereas if you have an appointment you've got to go to the appointment, you've got to hold the appointment, whatever. This is brilliant! Parent 1

Parents also spoke about their ability to engage in the intervention at their own pace; the self-help intervention allows parents to have control over the delivery of the intervention in a way that they may not be able to if delivered by a therapist. This allows parents to spend more time concentrating on a certain strategy and implementing it. Parents hinted this made the intervention effective. The flexibility of the intervention was also discussed in terms of their ability to return to an aspect of the intervention should they need to, because it was initially unsuccessful, or to refresh their memory and skills.

If you're in a parenting group you can forget things that have been said, or strategies, whereas in the book, it's there and you can go back to those strategies and re-read them if you want to and refresh. Parent 11

Parents who were faced with barriers that prevented them from engaging in the self-help intervention also displayed appreciation for its flexibility. They discussed their ability to return to the intervention at a more convenient time. If parents are enrolled in a therapist-led intervention and situational barriers prevent their ability to attend or engage, they may be unable to return again at a later date.

In a few weeks when things calm down, I'm going to look at it. That's what's good I suppose, it's something that, I've got it now, so I can just keep it and look at it when things aren't like what they are now. Parent 4

However, parents also discussed how the DVD that accompanied the self-help manual did not share the same levels of flexibility. Consequently, since the flexibility of the self-help manual was something that parents spoke about positively, parents had less complimentary views of the DVD.

I found the book easier because you can actually you know, sit in the garden with it or even sit in bed, where with the DVD you've got to be sat at the telly or at the computer which you know, it's not as flexible. Parent 5

\section{The self-help intervention vs. traditional formats:}

The solo nature of the self-help intervention

Parents had differing views on how the intervention compared to therapist-led interventions. As discussed above, parents acknowledged the flexibility of the intervention. However, when asked about how the self-help intervention compares, parents also missed the benefit of having input from other people. Some parents discussed the importance of having someone that they can go to during the programme for reassurance, motivation or queries.

I think it would be good for there to be some form of support base for parents so they can perhaps seek some clarification. Parent 9

One parent also discussed that she felt that she learnt a lot from other parents and missed the group-based format that traditional parenting interventions might offer. Sharing tried and tested techniques with other parents in similar positions might be a valuable aspect of intervention that is absent from self-help versions.

...because you're in a group and there are other people in your situation and you learn a lot from other parents, and you're not just reading a book. Parent 5

Some parents went on to say that the intervention could be improved by having someone there that they could approach should they need to seek advice, motivation or encouragement.

There needs to be some kind of support for parents in case you're faced with a situation where you are trying all of these strategies over and over and it's just not doing anything; 
whether that's because they are quite doing it right, or if it's because their child has more complex needs. Parent 8

\section{Subtheme: 'It's like having a therapist in a book'} However, two of the parents who were interviewed felt the self-help book was sufficient. They felt that the content of the book was self-explanatory and for this reason did not need to seek clarification. When asked how the self-help book could be improved, one of these parents suggested that she would prefer another book to come out that may help her with new issues that might present as her child approaches secondary school age.

. it is very easily explained, you knew straight away if you tried a certain thing and it didn't work then you knew you weren't ready to do that step, and you know, reverted to the previous chapter. Parent 1

\section{Trying to complete the intervention: barriers to engagement in the home environment}

Parents differed in the extent to which they actively engaged in the intervention; this seemed dependent on ability to overcome barriers preventing engagement. Parents acknowledged that there were often distractions (e.g. household chores and child care) at home that prevented them from engaging in the intervention. Consequently, trying to find time to sit down and read the book was sometimes difficult. One parent went on to say that attendance at a parenting group would remove household distractions ensuring time to dedicate to the intervention.

I think it's easy to get tied up with things at home. . and you're going on a course so you're not at home, you're not suddenly, 'Oh the washing needs doing'. Parent 3

Another key issue raised by parents was that other children in the house often prevented them from being able to engage in the intervention strategies. Parents discussed that they wanted time to engage with their child with ADHD on a one-to-one basis, but this was prevented because other children in the house also wanted to be involved. Parents also sometimes referred to the fact that more children in the house meant that their household demands are increased, and they struggled to put time aside to read and engage with the manual.

I have a family with four children and I didn't have time to sit down with just one child and play the game without the others wanting to participate...I got them all together [to play the games], they still just messed about. Parent 4

Parents also discussed how the behaviour of their child with ADHD at times acted as a barrier to engagement. The extent to which this occurred varied from some parents being unable to implement some of the play and memory games at a specific time because of their child's behaviour, commenting that it 'wasn't the right time' for their child. Parents discussed how their child's demanding behaviours take up all their time in the evening, and their children do not go to bed until late at night. Because parents have other things that they need to get done in the day, their child's complex and demanding behaviours in the evening mean that they are unable to sit down and actively engage in the intervention.
I have him 24 hours a day other than when he's at school, he doesn't go to bed until gone 11 every night, so I never have an evening to myself and he's pretty hard work. . I just feel like when he's gone to bed, it's half 10, I'm shattered. I don't have time to read anything. Parent 3

\section{Earlier access to help}

The majority of parents in this sample said that they would have preferred to have been offered this intervention at an earlier point in their child's care. They reflected on how they felt unsure of what to do when they received the diagnosis for their child.

But I think the learning that can be gained from this book, as early as possible, when you realise you have got a problem, the earlier the better for you and the child. Parent 9

In a similar vein, parents felt that they would have got more from the intervention and that the strategies in the book would have been more useful when their child was younger. Whilst parents were positive about the intervention acting as a reminder of things they had learnt before, because self-help intervention was a repetition of strategies they had previously encountered, they would have liked to have been offered the book earlier. One parent commented that the relationship between her and her child had become more strained as the child had got older, and hence, they would have preferred having it sooner. Another parent felt that because their child was older, some of his behaviours are now more difficult to change; they would have got more out of the book if they had received it earlier in their child's care.

In the very beginning we didn't know what was wrong; we really, really struggled with what to do with him. Now he's 10 , and he's quite mature for his age, so I feel that a lot of his behaviours are his ways, in the beginning when we didn't know what to do, it would have been a lot better for us. Parent 8

\section{Structured questions}

In response to the structured questions, all of the parents in the sample would have recommended this intervention to a friend, would have liked to have been offered it as part of their usual care from their clinician, and thought that the intervention should be routinely offered to parents when they access care for their child with ADHD. However, one parent also commented that there is a need to ensure that clinicians only offer the interventions to parents who could handle it and also stated that she would only offer the intervention to a friend if they were the parent of a single child. This same parent had also discussed involvement of siblings as a barrier to intervention (as described in the 'trying to complete the intervention: barriers to engagement in the home environment' theme above), and that additional support with involvement with siblings was important. In addition, one parent commented that the intervention should be routinely offered to parents but at a much earlier stage in their child's care.

\section{Discussion}

The aim of this study was to explore participant experience of the self-help version of the NFPP delivered in the context of a small-scale RCT. Overall, parents described 
positive views of the intervention, but differed in the extent to which they used it. Some reported an active involvement, by reading the entire manual and implementing as many strategies as they could. Other parents reported reading some of the book, but situational barriers preventing their ability to complete the self-help intervention.

All of the parents in this sample were motivated to participate in the study because they wanted to increase their knowledge of ADHD and behavioural strategies. Whilst this may not be a representative view of all parents of children with ADHD, it is clear that some parents in the healthcare system are looking for new strategies they can learn to help their child. It is important that there are accessible resources that can be offered to parents when they feel that they most need it. Since information provided on the internet may be variable in terms of its quality and accuracy (Kisely, Ong, \& Takyar, 2003), self-help manuals or psychoeducation from reputable sources could be a beneficial consideration for services, especially in situations where providing face-to-face interventions is problematic.

Parents acknowledged the flexibility of the intervention in terms of their ability to complete the intervention at home, and at a place and time most convenient to them. This supports the ability of self-help interventions to overcome practical barriers to accessing parenting interventions such as the inconvenient location of services, transport and child-care issues (Owens et al., 2002). Further, parents discussed benefits of the intervention for their parenting behaviour and increased empathy for their child's difficulties which might underlie challenging behaviour. Mostly, parents reported engaging in more positive parenting behaviours, such as engaging their child in play, the use of visual cues to aid child organisational skills and visual reward charts. Such strategies may be particularly beneficial for children with ADHD, who display hypersensitivity to social reinforcement such as praise and attention from parents (Kohls, Herpertz-Dahlmann, \& Konrad, 2009). Self-help interventions could provide a flexible approach to intervention delivery and could be particularly useful whilst physical distancing prevents face-to-face access to ADHD services (Cortese et al., 2020). However, whilst some parents acknowledged the flexibility of the intervention and the possibility of returning to the intervention at more convenient times, it is possible that some parents may not return to the intervention.

Barriers to the self-help intervention were also evident. The self-taught nature of the intervention in the home environment means that distractions are likely, particularly given that homes of children with ADHD might be more unstructured than homes of typically developing children, and the high heritability rates of ADHD (Johnston, Mash, Miller, \& Ninowski, 2012; Sonuga-Barke, Daley, \& Thompson, 2002). Parents mentioned that the presence of siblings, being distracted by other household chores or being preoccupied with adverse child behaviour as factors that prevented them from adhering to the selfhelp intervention. Parental motivation is likely associated with the perception of intervention barriers (Nock \& Photos, 2006) and has been highlighted as a key issue in qualitative studies exploring barriers to parenting interventions for disruptive behaviour (Koerting et al., 2013). If healthcare services consider self-help interventions, brief therapist input over the telephone could help to motivate parents or help them to identify ways to overcome barriers and improve intervention adherence (Nock \& Kazdin, 2005). Indeed, effect sizes for self-help interventions are larger when supplemented with brief therapist input (Tarver, Daley, Lockwood, et al., 2014). When delivered without therapist input, self-help interventions may provide most benefit to motivated parents, or parents who have recently received a diagnosis for their child (based on 'earlier access to help' theme). Self-help interventions could be offered and delivered as part of a stepped care approach to treatment, to cases of less severe behavioural problems or whilst families are on waiting lists. However, future RCTs of self-help interventions would need to confirm these hypotheses with moderation analyses.

One parent in this study also described how groupbased parenting interventions offer parents the opportunity to meet and engage with other parents. The ability to develop support networks, and learn from other parents, has been identified as an important aspect for parents accessing parent interventions for ADHD (Smith et al., 2015; Taylor et al., 2015). The solo nature of self-help interventions may limit parent ability to seek support and knowledge from other parents. Supplementation of self-help interventions with online discussion forums could help to overcome this. However, conversations would require careful moderation and should be optional; some parents report feelings of isolation and stigma as key barriers in accessing group-based therapist-led parent interventions (Prinz \& Sanders, 2007; Smith et al., 2015).

A final consideration for healthcare services is that parents felt that the self-help intervention would have been more beneficial if it was offered earlier in their child's care. Indeed, the creators of the NFPP, amongst others, argue that early and more targeted interventions are necessary to ensure better long-term outcomes for children with ADHD (Sonuga-Barke, Thompson, Abikoff, Klein, \& Brotman, 2006). Evaluation of the self-help intervention in families with a preschool child with ADHD is an important area for future research.

The findings of this study should be considered in light of some limitations. First, although attempts were made to interview all parents who received the self-help intervention, parents who dropped out of the study or those who could not be contacted were not interviewed. Since parents may be more likely to drop out if they had unacceptable views of the intervention or experienced situational barriers or low mood, the analysis may be missing their vital viewpoints. It is also of note that participants in the RCT, and consequently those in the nested qualitative study, tended to be highly educated. Further, clinicians were asked not to refer parents into the RCT whom they felt would be unable to complete the self-help intervention (e.g. due to mental health difficulties). The analysis presented here would therefore miss the important views of such parents. Consequently, the findings of this study may not generalise beyond this small selective sample. Second, for practicality reasons, interviews had to be conducted over the telephone. It has been argued that face-to-face interviews allow for a more natural conversation with visual cues that may encourage participants to elaborate further and provide more detailed data (Gubrium \& Holstein, 2001). However, since face-to-face interviews were not practically 
possible in this study, telephone interviews are seen as an acceptable alternative. Third, the interviews and data analysis were conducted by the same researcher who was also involved in recruitment and data collection during the RCT. Social desirability bias is a possibility in this study, although parents were asked to answer questions as honestly as possible. Confirmation bias is another possibility. However, the wider research team were involved in the analysis and interpretation of data to reduce risk of the lead researcher interpreting data to fit study hypotheses.

To our knowledge, this study is one of the first studies to use qualitative methodology to explore participants views of self-help parenting interventions for parents of children with ADHD. Given the number and range of barriers that can impact access and adherence to traditional therapist-led intervention, better exploration of the efficacy and acceptability of self-help intervention is warranted. This study revealed a number of key implications for researchers and healthcare professionals in the field. First, although children in this study were accessing treatment from healthcare services for ADHD, all parents described a desire to obtain more knowledge about ADHD and management. Self-help parent interventions may provide a low-cost, low-intensity option to provide parents with information when they feel they need it. This is especially true when parents first receive a diagnosis for ADHD. Second, whilst parents generally described favourable views of the intervention, barriers to its implementation were evident. Parents may benefit from some brief therapist input to help them overcome or plan for barriers they are likely to face when implementing interventions independently at home. Examples might include encouraging parents to timetable in time to engage in the intervention, or supporting parents with the inclusion of siblings in intervention components. Future research should explore participant acceptability of NFPP-SH, in addition to other self-help parenting programmes, when supplemented with brief therapist input.

\section{Acknowledgements}

This study was funded by National Institute for Health Research (NIHR) Collaboration for Leadership in Applied Health Research and Care - Nottinghamshire, Derbyshire, Lincolnshire (CLAHRC-NDL) and the Institute of Mental Health at the University of Nottingham. D.D. is a co-author of the published selfhelp version of the New Forest Parenting Programme. The remaining authors have declared that they have no competing or potential conflicts of interest.

\section{Ethical information}

This study received ethical approval from the NHS Research Ethics Committee (REC ref: 12/EM/0200). Full interview data not available for ethical reasons. All participants provided informed consent for their involvement in this study. All participants provided consent for publication in peer-reviewed journals.

\section{Correspondence}

Joanne Tarver, School of Psychology, College of Health and Life Sciences, Aston University, Aston Triangle, Birmingham B4 7ET, UK; Email: j.tarver@aston.ac.uk

\section{Supporting information}

Additional Supporting Information may be found in the online version of this article:

Appendix S1. Interview schedule.

\section{References}

Braun, V., \& Clarke, V. (2006). Using thematic analysis in psychology. Qualitative Research in Psychology, 3, 77-101.

Braun, V., \& Clarke, V. (2013). Successful qualitative research: A practical guide for beginners. London: SAGE Publications Inc.

Braun, V., \& Clarke, V. (2014). What can "thematic analysis" offer health and wellbeing researchers? International Journal of Qualitative Studies on Health and Well-Being, 9, 26152.

Campbell, M. (2000). Framework for design and evaluation of complex interventions to improve health. BMJ, 321(7262), 694-696.

Cortese, S., Asherson, P., Sonuga-Barke, E., Banaschewski, T., Brandeis, D., Buitelaar, J., ... \& Simonoff, E. (2020). ADHD management during the COVID-19 pandemic: Guidance from the European ADHD Guidelines Group. The Lancet Child \& Adolescent Health, 4, 412-414.

Daley, D., \& O'Brien, M. (2013). A small-scale randomized controlled trial of the self-help version of the New Forest Parent Training Programme for children with ADHD symptoms. European Child \& Adolescent Psychiatry, 22, 543-552.

Daley, D., Tarver, J., \& Sayal, K. (2020). Efficacy of a self-help parenting intervention for parents of children with Attention Deficit Hyperactivity Disorder in adjunct to usual treatment Small scale randomised Controlled Trial. Child: Care, Health and Development, 47, 269-280. https://doi.org/10.1111/cc h. 12825

Dose, C., Hautmann, C., Buerger, M., Schuermann, S., Woitecki, K., \& Doepfner, M. (2017). Telephone-assisted self-help for parents of children with attention-deficit/hyperactivity disorder who have residual functional impairment despite methylphenidate treatment: A randomized controlled trial. Journal of Child Psychology and Psychiatry, and Allied Disciplines, 58, 682-690.

Foster, E.M., Johnson-Shelton, D., \& Taylor, T.K. (2007). Measuring time costs in interventions designed to reduce behavior problems among children and youth. American Journal of Community Psychology, 40, 64-81.

Goldberg, D.P., \& Williams, P. (1988). A user's guide to the General Health Questionnaire. London: NFER-Nelson.

Gubrium, J., \& Holstein, J. (2001). Handbook of interview research. Thousand Oaks, CA: SAGE Publications Inc.

Ise, E., Kierfeld, F., \& Döpfner, M. (2015). One-year follow-up of guided self-help for parents of preschool children with externalizing behavior. The Journal of Primary Prevention, 36, 3340.

Johnston, C., Mash, E.J., Miller, N., \& Ninowski, J.E. (2012). Parenting in adults with attention-deficit/hyperactivity disorder (ADHD). Clinical Psychology Review, 32, 215-228.

Kazdin, A.E., \& Wassell, G. (2000). Predictors of barriers to treatment and therapeutic change in outpatient therapy for antisocial children and their families. Mental Health Services Research, 2, 27-40.

Kisely, S., Ong, G., \& Takyar, A. (2003). A survey of the quality of web based information on the treatment of Schizophrenia and attention deficit hyperactivity disorder. Australian \& New Zealand Journal of Psychiatry, 37, 85-91.

Koerting, J., Smith, E., Knowles, M.M., Latter, S., Elsey, H., McCann, D.C., ... \& Sonuga-Barke, E.J. (2013). Barriers to, and facilitators of, parenting programmes for childhood behaviour problems: A qualitative synthesis of studies of parents' and professionals' perceptions. European Child \& Adolescent Psychiatry, 22, 653-670.

Kohls, G., Herpertz-Dahlmann, B., \& Konrad, K. (2009). Hyperresponsiveness to social rewards in children and adolescents with attention-deficit/hyperactivity disorder (ADHD). Behavioral and Brain Functions, 5, 20. 
Lange, A.-M., Daley, D., Frydenberg, M., Houmann, T., Kristensen, L.J., Rask, C., .. . \& Thomsen, P.H. (2018). Parent training for preschool ADHD in routine, specialist care: A randomized controlled trial. Journal of the American Academy of Child \& Adolescent Psychiatry, 57, 593-602.

Laver-Bradbury, C., Thompson, M., Weeks, A., Daley, D., \& Sonuga-Barke, E.J. (2010). Step by step help for children with ADHD: A self-help manual for parents. London: Jessica Kingsley.

Lewin, S., Glenton, C., \& Oxman, A.D. (2009). Use of qualitative methods alongside randomised controlled trials of complex healthcare interventions: Methodological study. BMJ, 339, b3496.

NICE (2018). Attention deficit hyperactivity disorder: Diagnosis and management. NICE guideline NG87. London: The British Psychological Society and the Royal College of Psychiatrists.

Nock, M.K., \& Kazdin, A.E. (2005). Randomized controlled trial of a brief intervention for increasing participation in parent management training. Journal of Consulting and Clinical Psychology, 73, 872-879.

Nock, M.K., \& Photos, V. (2006). Parent motivation to participate in treatment: Assessment and prediction of subsequent participation. Journal of Child and Family Studies, 15, 333346.

Owens, P.L., Hoagwood, K., Horwitz, S.M., Leaf, P.J., Poduska, J.M., Kellam, S.G., \& Ialongo, N.S. (2002). Barriers to children's mental health services. Journal of the American Academy of Child \& Adolescent Psychiatry, 41, 731-738.

Prinz, R.J., \& Sanders, M.R. (2007). Adopting a population-level approach to parenting and family support interventions. Clinical Psychology Review, 27, 739-749.

Shimabukuro, S., Daley, D., Thompson, M., Laver-Bradbury, C., Nakanishi, E., \& Tripp, G. (2017). Supporting Japanese mothers of children with ADHD: Cultural adaptation of the new forest parent training programme. Japanese Psychological Research, 59, 35-48.

Smith, E., Koerting, J., Latter, S., Knowles, M.M., McCann, D.C., Thompson, M., \& Sonuga-Barke, E.J. (2015).
Overcoming barriers to effective early parenting interventions for attention-deficit hyperactivity disorder (ADHD): Parent and practitioner views. Child: Care, Health and Development, 41, 93-102.

Sonuga-Barke, E.J.S., Barton, J., Daley, D., Hutchings, J., Maishman, T., Raftery, J., .. \& \& Thompson, M.J.J. (2018). A comparison of the clinical effectiveness and cost of specialised individually delivered parent training for preschool attentiondeficit/hyperactivity disorder and a generic, group-based programme: A multi-centre, randomised controlled trial of the New Forest Parenting Programme versus Incredible Years. European Child \& Adolescent Psychiatry, 27, 797-809.

Sonuga-Barke, E.J.S., Daley, D., \& Thompson, M. (2002). Does maternal ADHD reduce the effectiveness of parent training for preschool children's ADHD? Journal of the American Academy of Child \& Adolescent Psychiatry, 41, 696-702.

Sonuga-Barke, E.J.S., Thompson, M., Abikoff, H., Klein, R., \& Brotman, L.M. (2006). Nonpharmacological interventions for preschoolers with ADHD: The case for specialized parent training. Infants \& Young Children, 19, 142-153.

Tarver, J., Daley, D., Lockwood, J., \& Sayal, K. (2014). Are selfdirected parenting interventions sufficient for externalising behaviour problems in childhood? A systematic review and meta-analysis. European Child \& Adolescent Psychiatry, 23, 1123-1137.

Tarver, J., Daley, D., \& Sayal, K. (2014). Attention-deficit hyperactivity disorder (ADHD): An updated review of the essential facts. Child: Care, Health and Development, 40, 762-774.

Taylor, J.A., Valentine, A.Z., Sellman, E., Bransby-Adams, K., Daley, D., \& Sayal, K. (2015). A qualitative process evaluation of a randomised controlled trial of a parenting intervention in community (school) settings for children at risk of attention deficit hyperactivity disorder (ADHD). BMC Psychiatry, 15, 290 .

Accepted for publication: 29 April 2021 